\title{
One-Step Synthesis of $\mathrm{MoS}_{2} / \mathrm{WS}_{2}$ Layered
}

\section{Heterostructures and Catalytic Activity of Defective}

\section{Transition Metal Dichalcogenide Films}

\author{
John M. Woods ${ }^{1,2}$, Yeonwoong Jung ${ }^{*}{ }^{1,2}$, Yujun Xie ${ }^{1,2,3}$, Wen Liu ${ }^{2,4}$, Yanhui Liu ${ }^{1,3}$, Hailiang \\ Wang $^{2,4}$, and Judy J. Cha $a^{1,2,3}$
}

1 Department of Mechanical Engineering and Materials Science, Yale University, New Haven, Connecticut 06511, United States

${ }^{2}$ Energy Sciences Institute, Yale University West Campus, West Haven, Connecticut 06477, United States

${ }^{3}$ Center for Research on Interface Structure and Phenomena, Yale University, New Haven, CT 06511, USA

${ }^{4}$ Department of Chemistry, Yale University, New Haven, CT 06511, USA

* Present address: Nanoscience Technology Center, Department of Materials Science and Engineering, University of Central Florida, Orlando, Florida 32826, USA

CORRESPONDING AUTHOR FOOTNOTE:

*Corresponding author. E-mail: judy.cha@yale.edu

Telephone number: +1 (203) 737-7293, Fax number: (203) 432-6775 
a)

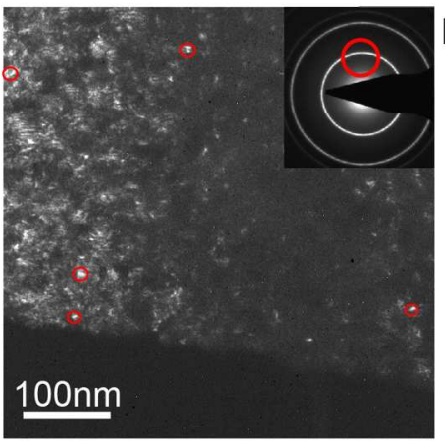

d)

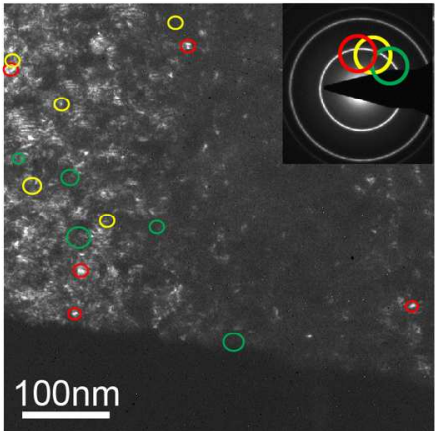

b)

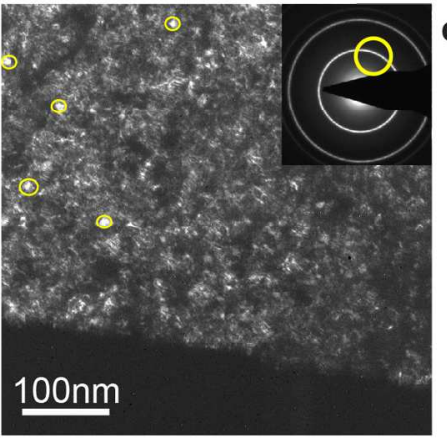

e)

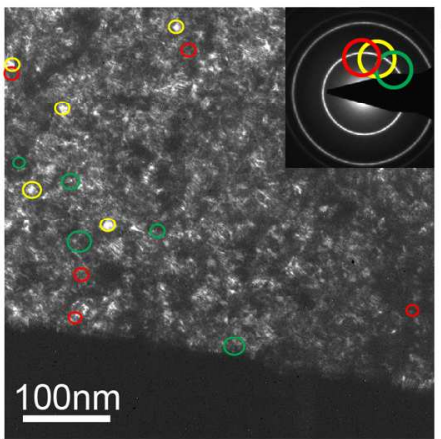

c)

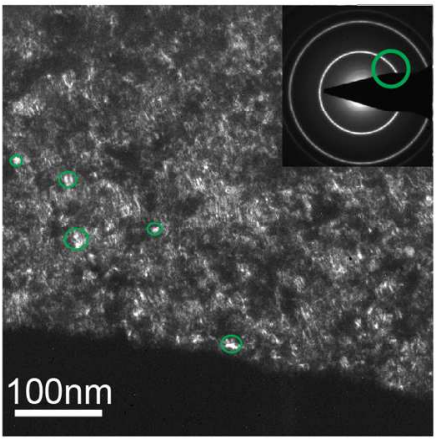

f)

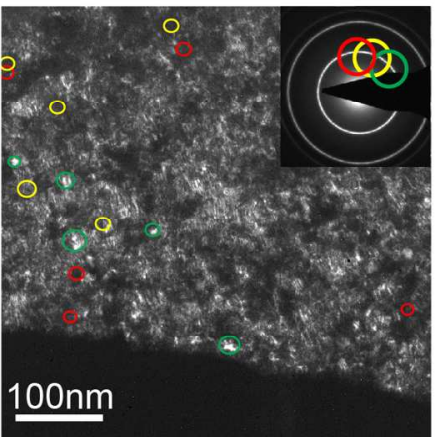

Figure S1. Dark field (DF) TEM images of $\mathrm{MoS}_{2} / \mathrm{WS}_{2}$ heterostructure flake. Images with five brightest spots circled shown for the objective aperture located at 12 O'clock (a), 1 O'clock (b), and 2 O'clock (c). The inset shows the location of the objective aperture. (d-f) Composite location of bright spots for 12 O'clock (red), 1 O'clock (yellow), \& 2 O'clock (green) overlaid on DF image for 12 O'clock (d), 1 O'clock (e), \& 2 O'clock (f). Due to the sample's polycrystalline nature, the bright spots for one aperture location do not match up with the location of bright spots for other aperture locations 
a)
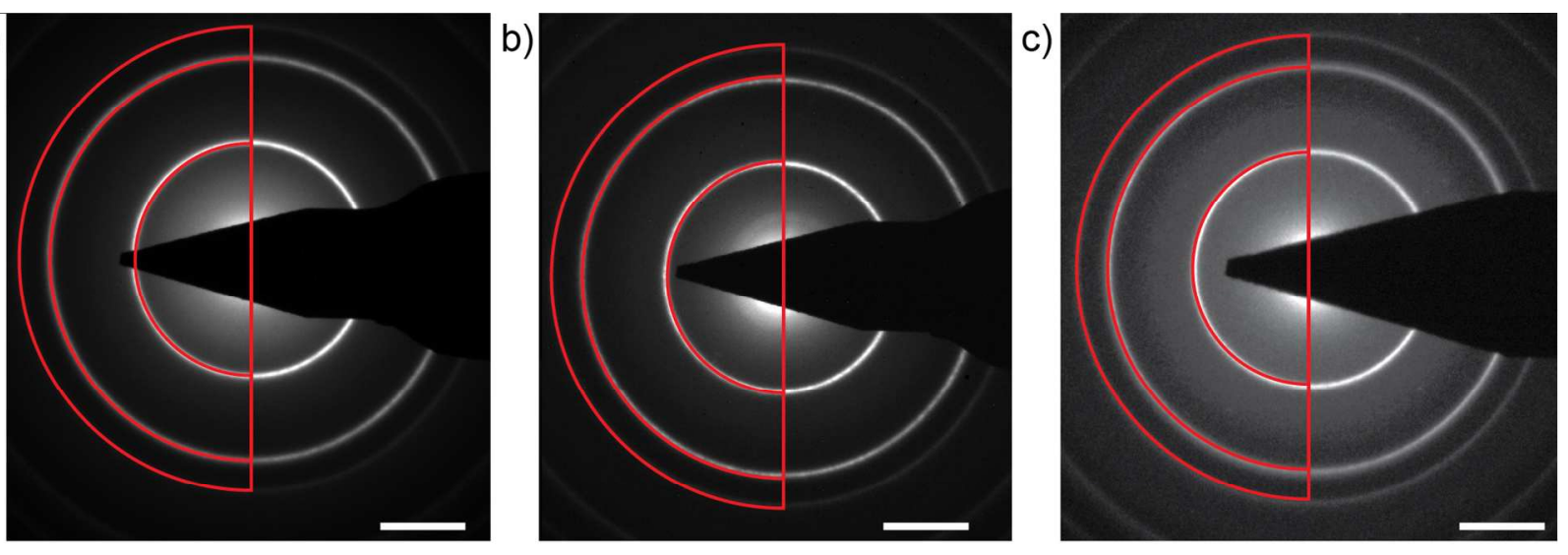

Figure S2. Selected area diffraction patterns for $\mathrm{MoS}_{2}$ (a), $\mathrm{WS}_{2}$ (b), and $\mathrm{MoS}_{2} / \mathrm{WS}_{2}$ heterostructure (c). All scale bars are $101 / \mathrm{Gm}$. Red half-circle overlays to show similar size of diffraction rings. 

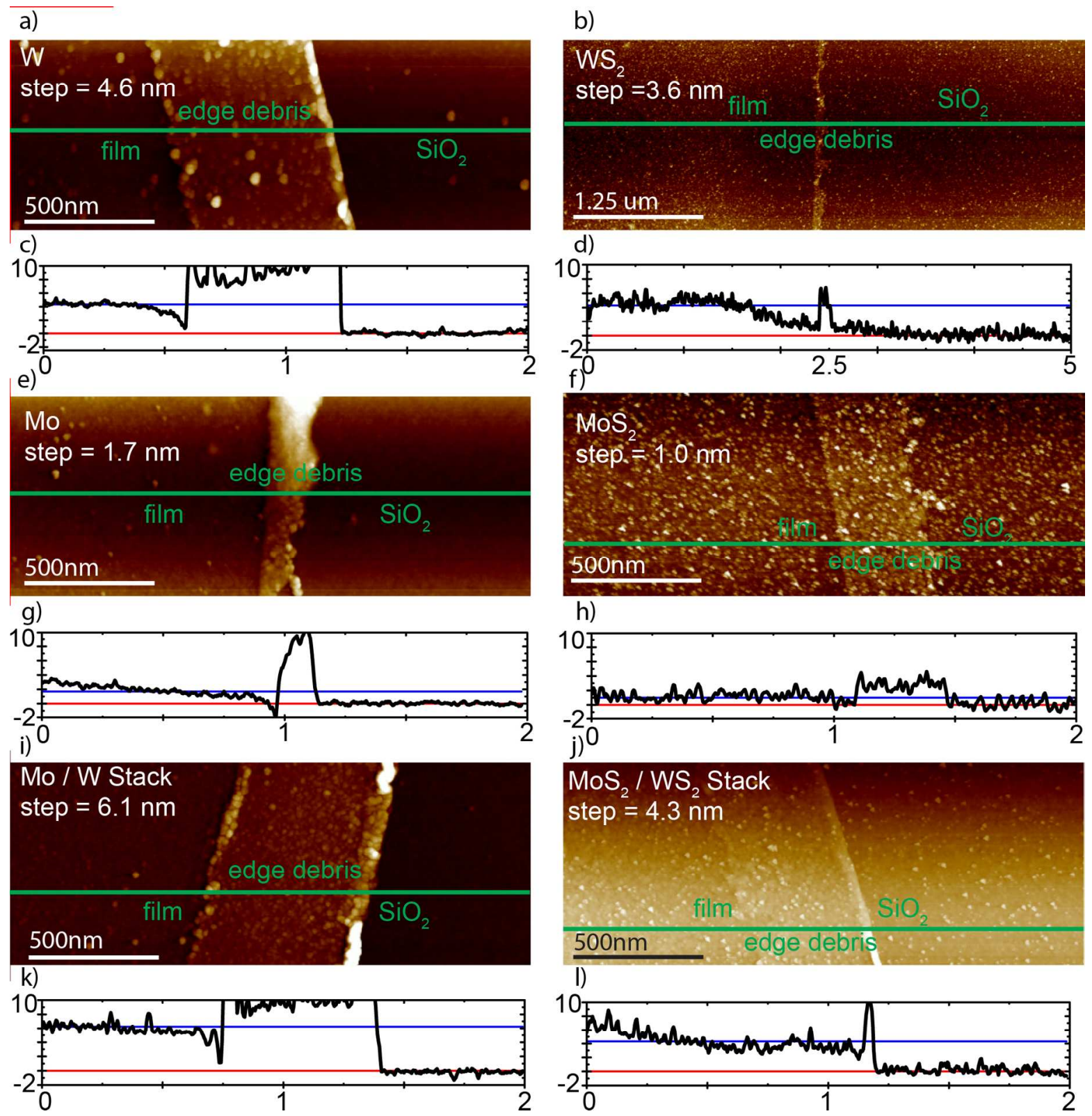

Figure S3. Atomic force microscopy images with corresponding step profiles for W (a,c), Mo $(\mathrm{e}, \mathrm{g}), \mathrm{Mo} / \mathrm{W}$ metal stack $(\mathrm{i}, \mathrm{k})$, and corresponding chalcogenides after sulfurization, $\mathrm{WS}_{2}(\mathrm{~b}, \mathrm{~d})$, $\mathrm{MoS}_{2}(\mathrm{f}, \mathrm{h})$, and $\mathrm{MoS}_{2} / \mathrm{WS}_{2}(\mathrm{j}, 1)$. The stated value for the step is the average of 3 profiles. For plots of step profiles the $\mathrm{x}$-axis is in $\mu \mathrm{m}$, the $\mathrm{y}$-axis is in $\mathrm{nm}$, and the scan was taken along the green line on the corresponding image. Lines are drawn for the height of the film (blue), and $\mathrm{SiO}_{2}$ (red). 

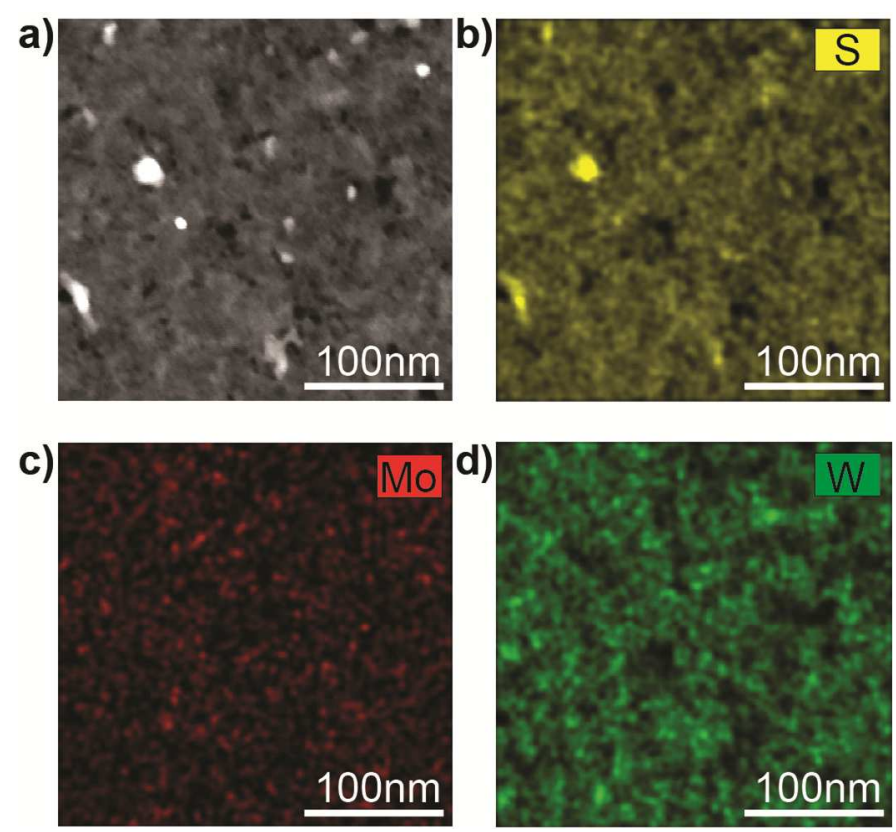

Figure S4. HAADF STEM-EDS mapping of patterned heterostructure. (a) HAADF STEM image of region of heterostructure flake. (b-d) STEM-EDS maps showing uniform spatial distribution of (b) S, (c) Mo, and (d) W. 

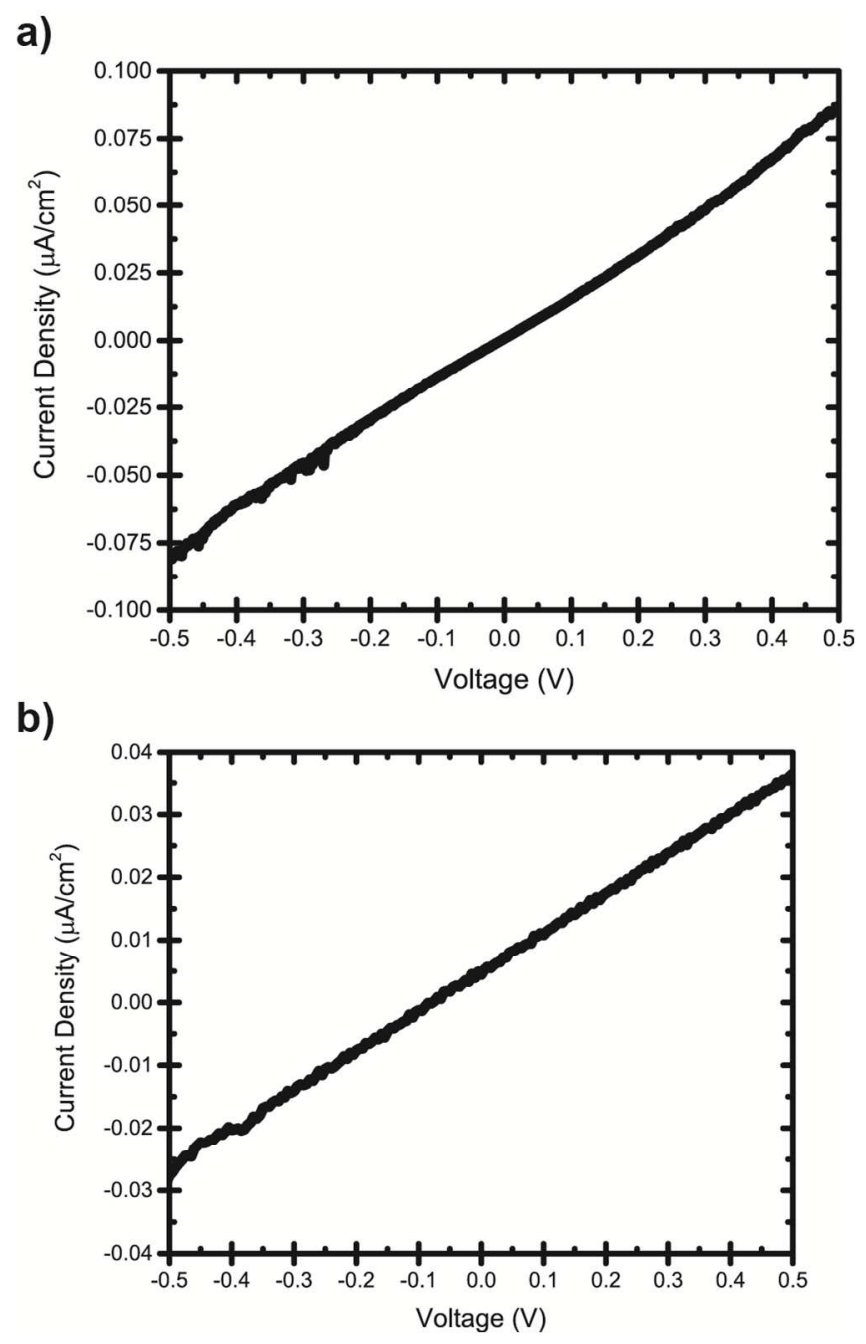

Figure S5. Electrical behavior of single species (a) $\mathrm{MoS}_{2}$ and (b) $\mathrm{WS}_{2}$ grown from $\sim 1 \mathrm{~nm}$ seed layers. 


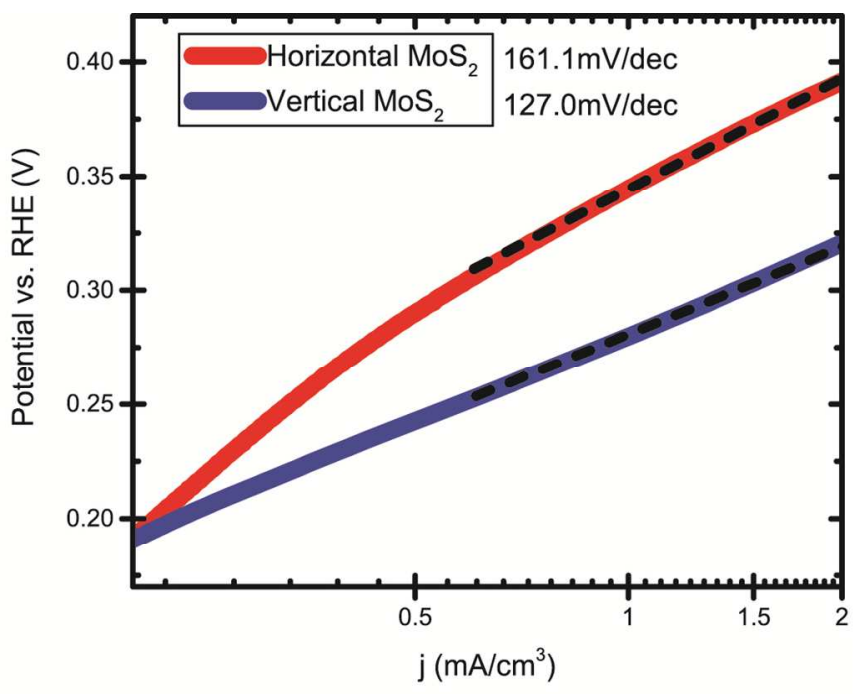

Figure S6. Tafel plots for horizontally and vertically oriented $\mathrm{MoS}_{2}$ films. 